\title{
A mode filter for plasma waves in the Hall-MHD approximation
}

\author{
C. Vocks ${ }^{1}$, U. Motschmann ${ }^{2}$, K.-H. Glassmeier \\ Institut für Geophysik und Meteorologie, Technische Universität Braunschweig, Mendelssohnstrasse 3, D-38106 Braunschweig, Germany \\ ${ }^{1}$ Now at Max-Planck-Institut für Aeronomie, Max-Planck-Strasse 2, D-37191 Katlenburg-Lindau, Germany \\ ${ }^{2}$ Also at the German Aerospace Center (DLR), Institute for Planetary Exploration, D-12484 Berlin, Germany
}

Received: 25 August 1998 / Revised: 10 November 1998 / Accepted: 20 November 1998

\begin{abstract}
A filter method is presented which allows a qualitative and quantitative identification of wave modes observed with plasma experiments on satellites. Hitherto existing mode filters are based on the MHD theory and thus they are restricted to low frequencies well below the ion cyclotron frequency. The present method is generalized to cover wave modes up to the characteristic ion frequencies. The spectral density matrix determined by the observations is decomposed using the eigenvectors of the linearized Hall-MHD equations. As the wave modes are dispersive in this formalism, a precise determination of the $k$-vectors requires the use of multi-point measurements. Therefore the method is particularly relevant to multi-satellite missions. The method is tested using simulated plasma data. The Hall-MHD filter is able to identify the modes excited in the model plasma and to assign the correct energetic contributions. By comparison with the former method it is shown that the simple MHD filter leads to large errors if the frequency is not well below the ion cyclotron frequency. Further the range of validity of the linear theory is examined rising the simulated wave amplitudes.
\end{abstract}

Key words. Magnetospheric physics (MHD waves and instabilities; plasma waves and instabilities).

\section{Introduction}

Satellite measurements of plasma characteristics such as the magnetic field, the velocity and density of the plasma or plasma composition are important to understand the different plasma wave modes which may occur. However, wave analysis requires further information and special tools in order to be able to identify which set of modes is contributing to observed wave features.

Correspondence to: C. Vocks
Analysis of the measurements has therefore the aim to identify plasma wave modes contributing to the signal, to decide if the signal consists of more than one mode, and to determine the contributions of the different wave modes to the total wave energy. This kind of analysis requires a filter to perform "pattern recognition", that is detecting the different kinds of modes that may propagate in the plasma and to determine their contributions to the wave energy.

Such mode filters do already exist for the magnetohydrodynamics (MHD) approximation and are based on the following principle (Glassmeier et al., 1995): the spectral amplitudes of plasma parameters such as the magnetic field vector, the mass density, and the velocity vector are collected in a state vector. The set of all state vectors forms a state space. The spectral amplitudes of the different possible linear wave modes of the underlying plasma model are collected in vectors in the same way. These vectors describe the polarizations of the modes and are therefore called polarization vectors. These polarization vectors form a basis of the state space (Samson and Olson, 1980; Samson, 1983). Any measured state vector may be decomposed in this basis in a unique way. Thus, it is possible to determine the contributions of the different wave modes to the measured signal as long as the wave modes to be analysed can be treated as linear perturbations.

In case of the MHD the state space is 7-dimensional. Disregarding entropy variations reduces the number of dimensions to six. The effect of this neglect of the entropy coordinate is discussed later in this paper. The polarization vectors of the fast, Alfvén and slow mode, each forward and backward propagating, then form a basis of the state space. The mode filter using this basis allows the investigation of any measured MHD signal for the contributions of these modes. It should be mentioned that due to the linear dispersive behaviour of the MHD wave modes the polarization vectors and phase velocities of the MHD wave modes are independent of the wave number and frequency. This does not hold for the Hall-MHD mode filter to be described here. 
The MHD formalism is a plasma model which neglects the ion inertia. Therefore it is only applicable to frequencies well below the ion cyclotron frequency $\Omega_{i}$ (Spitzer, 1962; Stix, 1962; Siscoe, 1983; Swanson, 1989). The aim of the work presented here is to remove this limitation and to develop a mode filter which is also applicable at higher frequencies. As the mode filter is based on the use of polarization vectors resulting from a particular plasma model the filter is model-dependent. Expansion of the mode filter concept to higher frequencies thus requires a different plasma model, applicable to higher frequencies.

Multi-fluid theories are plasma models whose validity does not exhibit any frequency limit (Holter et al., 1973). However, expansion of the mode filter concept by employing a multi-fluid theory leads to a very high dimension of the state space. In the case of a two-fluid theory it was found to be 13-dimensional. Therefore, it seems to be of great advantage to use a plasma model that is not limited to low frequency $\omega \ll \Omega_{i}$ and that does not increase the dimension of the state space in this way. An important step in expanding the frequency range that fulfills this requirement is the change from the MHD to the Hall-MHD.

The Hall-MHD mode filter presented here provides a method for analysing the energetic composition of the measured signal and allows a determination of the wave modes contributing to it. This is performed by a joint interpretation of the data of a single satellite on the plasma parameters magnetic field, density and velocity. This kind of interpretation can be regarded as an "intraspacecraft-analysis" of the data from different instruments onboard the satellite. Since the Hall-MHD wave modes are dispersive, the application of the Hall-MHD mode filter requires not only the determination of the plasma background parameters, but also of the wave number $k$. The wave analyzer of Motschmann et al. (1996) provides this $k$ using an "inter-spacecraft analysis" of the magnetic field data of four satellites, but it cannot determine the composition of the measured signal with respect to the different wave modes. Thus, the Hall-MHD mode filter expands the frequency range in comparison with standard MHD theory and completes the analysis of multi-spacecraft data.

\section{The model}

Hall-MHD has been extensively studied, e.g. Hassam and Huba (1988) and Huba (1995), and allows the description of the dispersion characteristics of the different plasma wave modes for small as well as large wave numbers. The Hall-MHD formulation treats the plasma as a single fluid much as the MHD does, but it considers the finite ion inertia. In this way it is possible to describe waves with frequencies up to $\omega \approx \Omega_{i}$. Because the model still neglects the electron mass, it is limited to frequencies well below the lower hybrid frequency: $\omega \ll \omega_{L H}$. The use of Hall-MHD instead of MHD will extend the frequency range in which the mode filter can operate.
To develop the Hall-MHD mode filter in analogy to the MHD mode filter as described by Glassmeier et al. (1995), it is necessary to determine the polarizations of the wave modes. To do so, we start with the basic equations of the ideal Hall-MHD:

$$
\begin{aligned}
& \frac{\partial \vec{B}}{\partial t}=\nabla \times(\vec{v} \times \vec{B})-\frac{1}{e N} \nabla \times(\vec{j} \times \vec{B}) \\
& \rho\left(\frac{\partial \vec{v}}{\partial t}+(\vec{v} \cdot \nabla) \vec{v}\right)=\vec{j} \times \vec{B}-\nabla p \\
& \frac{\partial \rho}{\partial t}+\nabla \cdot(\rho \vec{v})=0
\end{aligned}
$$

This set of equations is completed by:

$$
\begin{aligned}
& \nabla \cdot \vec{B}=0 \\
& \nabla \times \vec{B}=\mu_{0} \vec{j}
\end{aligned}
$$

These equations are nearly identical with the basic equations of the MHD, except for the Hall-term $\frac{1}{e N} \nabla \times(\vec{j} \times \vec{B})$ in Eq. (1). However, this term leads to important differences with respect to the MHD mode filter, because it gives rise to the nonlinear dispersive behaviour of the Hall-MHD plasma wave modes. The current density $\vec{j}$ can be eliminated using the Maxwell Eq. (5). The basic equations are linearized around the undisturbed state $\vec{B}^{0}, \rho^{0}, \vec{v}^{0}$ and $\vec{j}^{0}=0$. These background parameters are temporally and spatially constant, the plasma background system considered is homogenous. For the effect of an inhomogeneity see Kingsep et al. (1990) or Huba (1991). After the linearization a Fourier transformation is performed: $\nabla \rightarrow i k, \frac{\partial}{\partial t} \rightarrow-i \omega$.

The linearized, Fourier transformed equations for the disturbances $\vec{B}^{1}, \rho^{1}, \vec{v}^{1}$ and $\vec{j}^{1}$ are:

$$
\begin{aligned}
\omega \vec{B}^{1}= & -\vec{k} \times\left(\vec{v}^{0} \times \vec{B}^{1}\right)-\vec{k} \times\left(\vec{v}^{1} \times \vec{B}^{0}\right) \\
& +\frac{1}{e N^{0}} \vec{k} \times\left(\vec{j}^{1} \times \vec{B}^{0}\right) \\
\rho^{0} \omega \vec{v}^{1}= & \rho^{0}\left(\vec{v}^{0} \cdot \vec{k}\right) \vec{v}^{1}+i \vec{j}^{1} \times \vec{B}^{0}+c_{s}^{2} \vec{k} \rho^{1} \\
\omega \rho^{1}= & \left(\vec{v}^{0} \cdot \vec{k}\right) \rho^{1}+\rho^{0} \vec{k} \cdot \vec{v}^{1}
\end{aligned}
$$

where $v_{A}=\frac{B^{0}}{\sqrt{\mu_{0} \rho^{0}}}$ is the Alfvén speed and $c_{s}$ the sound
speed.

Eqs. (6)-(8) are rewritten in matrix form, using the coordinate system defined in Fig. 1.

The $x$-axis points into the direction of the wave vector $\vec{k}$, and the background magnetic field $\vec{B}^{0}$ lies in the $x-z$ plane, including an angle $\theta$ with the $x$-axis: $\vec{B}^{0}=$ $B^{0}(\cos \theta, 0, \sin \theta)^{T}$.

From Eq. (4) it follows that the $x$-component of the perturbation magnetic field vanishes: $B_{x}^{1}=0$. Equations (6)-(8) can be summarized introducing the state vector

$$
\vec{u}=\frac{1}{v_{A}}\left(v_{y}^{1}, \frac{B_{y}^{1}}{\sqrt{\mu_{0} \rho^{0}}}, v_{x}^{1}, v_{z}^{1}, \frac{B_{z}^{1}}{\sqrt{\mu_{0} \rho^{0}}}, \frac{c_{s} \rho^{1}}{\rho^{0}}\right)^{T}
$$




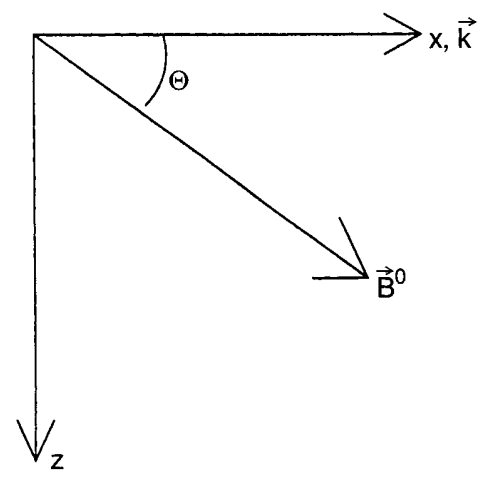

Fig. 1. The coordinate system for the Hall-MHD mode filter

This state vector and therefore the state space is 6-dimensional. It is the same state space as in case of the MHD mode filter. Thus, changing from the MHD to the Hall-MHD plasma model keeps the dimension of the state space unchanged. Rearranging the components of Eqs. (6)-(8) yields the following system of equations:

$k \mathbf{Z} \cdot \vec{u}=\omega \vec{u}$

The system matrix $\mathbf{Z}$ is defined as:

$\mathbf{Z}(k)=\left(\begin{array}{cccc}v_{x}^{0} & -v_{A} \cos \theta & 0 & 0 \\ -v_{A} \cos \theta & v_{x}^{0} & 0 & 0 \\ 0 & 0 & v_{x}^{0} & 0 \\ 0 & 0 & 0 & v_{x}^{0} \\ 0 & i k d v_{A} \cos \theta & v_{A} \sin \theta & -v_{A} \cos \theta \\ 0 & 0 & c_{S} & 0\end{array}\right.$

$\left.\begin{array}{cc}0 & 0 \\ -i k d v_{A} \cos \theta & 0 \\ v_{A} \sin \theta & c_{s} \\ -v_{A} \cos \theta & 0 \\ v_{x}^{0} & 0 \\ 0 & v_{x}^{0}\end{array}\right)$

where $d$ is given as $d=\frac{v_{A}}{\Omega}$. This $d$ can be identified as the ion inertial length: $d \stackrel{\Omega_{i}}{=} \frac{c}{\omega_{p i}}$. Eq. (10) can be written as an eigenvalue equation:

$\mathbf{Z} \cdot \vec{u}=\frac{\omega}{k} \vec{u}$

The eigenvalues correspond to the phase velocities of the different wave modes and the eigenvectors denote their polarizations. The system matrix $\mathbf{Z}$ is a Hermitian 6 by 6 matrix. This guarantees the existence of six real eigenvalues and six orthogonal eigenvectors. So it is always possible to calculate the phase velocities $v_{p h, i}$ and polarizations $\vec{e}_{i}$ of the six Hall-MHD wave modes.

The system matrix $\mathbf{Z}$ presented here depends not only on the direction of the wave propagation, given by the parameter $\theta$, but also on the wave number $k$. This dependence on $k$ is introduced by two elements of the matrix, which originate from the Hall-term in Eq. (1). Since this Hall-term is the only difference between the basic equations of MHD and Hall-MHD, the system matrix $\mathbf{Z}$ of the Hall-MHD mode filter and the corresponding matrix of the MHD mode filter differ only in these two elements. In the case of MHD they are missing, and the system matrix is independent of the wave number $k$, and so are their eigenvalues and eigenvectors. MHD exhibits a linear dispersion behaviour, and the polarizations of the MHD modes are constant. This is not the case for Hall-MHD. The polarizations of the modes depend on $k$, and the phase velocities are no longer constant. This is expected for a plasma model which is valid for frequencies $\omega \approx \Omega_{i}$ because phenomena like the ion cyclotron resonance (Gurnett et al., 1965) are impossible to describe with a linear dispersion.

In Hassam and Huba (1988) and Huba (1995) the dispersion characteristics and the eigenmodes of the Hall-MHD plasma model were discussed for small wave numbers $k v_{A} \ll \Omega_{i}$ as well as large ones $k v_{A} \gg \Omega_{i}$. To develop the mode filter, solutions of the eigenvalue Eq. (12) are required for any wave number $k$. The first step in solving this problem is the determination of the eigenvalues. This is performed by setting the characteristic polynomial of the system matrix $\mathbf{Z}$ equal to 0 . The resulting equation is a condition for the frequency $\omega$ and wave number $k$. Thus, the eigenvalue equation leads to the dispersion relation of the Hall-MHD, determination of the zeroes of the characteristic polynomial of the system matrix corresponds to the investigation of the dispersive nature of the plasma model.

The system matrix $\mathbf{Z}$ in Eq. (11) is 6-dimensional. Its characteristic polynomial has the degree 6 in $\omega$ and 8 in $k$. For polynomials of the degree 4 or less analytic formulae for their zeroes do exist, but for higher degrees no general analytic formula has been found. Thus, it seems impossible to give an analytic expression for the eigenvalues of $\mathbf{Z}$. In that case the eigenvalue equation must be solved numerically. However, we derived an analytic expression for the solutions $\omega(k)$ of the dispersion relation. The derivation of this formula is a little lengthy and is therefore summarized in the appendix. Here only the result is presented:

$$
\begin{array}{r}
\frac{\omega}{k}=v_{x}^{0} \\
\pm \sqrt{\frac{X 1}{3}+\frac{2}{3} \sqrt{-a} \cos \left(\frac{1}{3} \arctan \left(\sqrt{-1-\frac{4 a^{3}}{b^{2}}}\right)+\frac{2 m}{3} \pi\right)} \\
m=0,1,2
\end{array}
$$

where the following abbreviations have been introduced:

$$
\begin{aligned}
X 1 & :=c_{s}^{2}+v_{A}^{2}+\cos ^{2} \theta v_{A}^{2}+\cos ^{2} \theta d^{2} k^{2} v_{A}^{2} \\
X 2 & :=2 c_{s}^{2}+c_{s}^{2} d^{2} k^{2}+v_{A}^{2} \\
a & :=-X 1^{2}+3 \cos ^{2} \theta v_{A}^{2} X 2 \\
b & :=27 \cos ^{4} \theta c_{s}^{2} v_{A}^{4}+2 X 1^{3}-9 \cos ^{2} \theta v_{A}^{2} X 1 X 2
\end{aligned}
$$


This formula allows the determination of the eigenvalues without any numerical solution of a system of equations.

Figure 2 shows the dispersion characteristics of a plasma in its rest frame. The parameter $\beta$ is here defined as $\beta:=\left(c_{s}^{2} / v_{A}^{2}\right)$. A flow $\vec{v}^{0}$ would only lead to a Doppler shift $\omega \rightarrow \omega+k v_{x}^{0}$, as is shown in the appendix. The three forward propagating modes are plotted; altogether there are six dispersion branches.

For small wave numbers $k$, the plasma models of Hall-MHD and MHD coincide, since their only difference, the Hall-term in Eq. (6), contains a factor $k$. This can be seen in Fig. 2. For small $k$, the three modes display a linear dispersion, as expected for the MHD regime. The fast, Alfvén and slow mode can be identified. However at higher wave numbers, deviations from MHD behaviour occur. The fast mode exhibits a higher phase velocity $\omega / k$ and turns into a whistler mode, and the slow mode becomes an ion cyclotron wave. The Alfvén mode first stays near the ion cyclotron frequency $\Omega_{i}$ and then turns into a sound wave. In this paper we decide on the convention to name the branches fast mode, Alfvén mode and slow mode also beyond the MHD limit $\omega / \Omega_{i} \ll 1, k v_{A} / \Omega_{i} \ll 1$.

Using Eq. (13) it is possible to find six solutions $\omega$ for any given $k$. The converse, however, is not true. The plot in Fig. 2 is an example of this. For frequencies $\omega<\Omega_{i} \cos \theta$ there are three values of $k$ satisfying the dispersion relation. The backward propagating waves also provide three solutions, so that six solutions for $k$ can be identified for any $\omega<\Omega_{i} \cos \theta$. However, this is not the case for $\omega>\Omega_{i} \cos \theta$. For these $\omega$, Fig. 2 shows that there are only two solutions for $k$, so that there is a total of only four solutions for $k$. This is due to the slow mode/ion cyclotron wave not reaching these frequencies. In the case of a moving plasma the situation would be even more complicated, since the Doppler shift term $k v_{x}^{0}$ would lead to local extremes of the $\omega(k)$ - curves which prevents one from finding the inverse functions. Therefore, no functional dependence $k(\omega)$ can be given which is valid for all frequencies $\omega$.

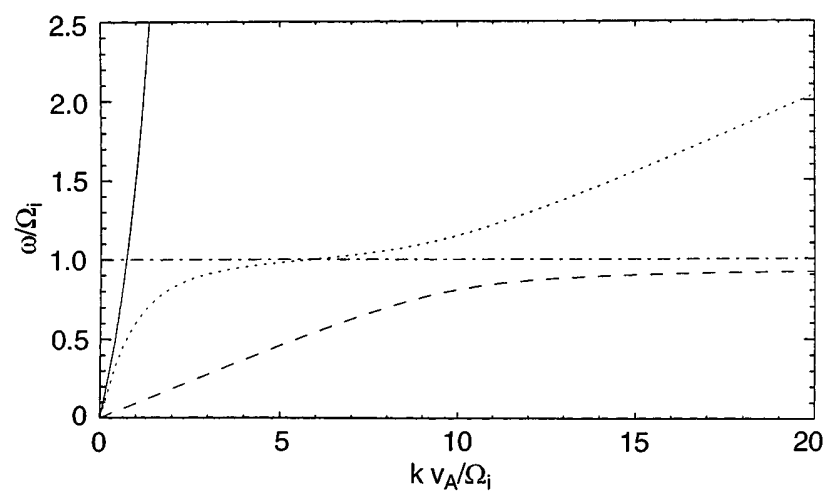

Fig. 2. The dispersion relations of the Hall-MHD. Plasma background parameters: $\theta=20^{\circ}, \beta=0.01, v_{x}^{0}=0$. In the low frequency limit $\left(\omega \ll \Omega_{i}\right)$ the branches correspond to the fast mode (solid line), Alfvén mode (dotted line) and slow mode (dashed line)
Due to the complicated structure of the solutions $\omega(k)$ presented in Eq. (13) we have not been able to find analytical expressions for their corresponding eigenvalues. However, numerical determination is straightforward.

Figure 3 displays the six components of the polarization vectors of the forward propagating modes as functions of the wave number for the same plasma background parameters as in Fig. 2. For any given $k$ it is thus not only possible to determine the frequencies of the modes, but also their polarizations. For the limit of small wave numbers $k \rightarrow 0$ the transition to the MHD can be studied. The only mode having components $B_{y}^{1}$ and $v_{y}^{1}$ is the Alfvén wave, and this mode does not have contributions from other components. Since both $B_{y}^{1}$ and $v_{y}^{1}$ carry the same phase factor $i$ in Fig. 3, the linear polarization of the Alfvén mode (dotted line) is reproduced in the correct manor. The other components $v_{x}^{1}, v_{z}^{1}, B_{z}^{1}$ and $\rho^{1}$ have no phase factors, so that the fast and slow mode are also linearly polarized.

For higher wave numbers, the modes change their polarizations. The fast mode (solid line) and the slow mode (dashed line) gain contributions from $B_{y}^{1}$ and $v_{y}^{1}$, and the Alfvén mode develops contributions from the other four components. Thus, the clear distinction between these modes in MHD is lost in the HallMHD framework. For very large wave numbers, the fast mode is dominated by contributions from $B_{y}^{1}$ and $B_{z}^{1}$. $B_{y}^{1}$ has the phase factor $i$, so this wave is elliptically polarized. The contributions from $B_{y}^{1}$ and $B_{z}^{1}$ are of the same size, the wave is even circularly polarized. The fast mode turns into a whistler mode. The slow mode which behaves in a similar way, contains mainly contributions from $v_{z}^{1}$ and $i \cdot v_{y}^{1}$ of the same magnitude, and it too changes into a circularly polarized wave. This is an ion cyclotron wave, as mentioned above in the discussion of the dispersion relation in Fig. 2. The Alfvén wave shows a different characteristic in the limit of large $k$. It consists mainly of variations in $v_{x}^{1}$ and $\rho^{1}$, and it turns into a sound wave.

We have found the eigenvalues of Eq. (12), i.e. we solved for the dispersion relation. We are further able to determine the eigenvectors corresponding to these eigenvalues, i.e. we can determine the polarizations of the different wave modes. Thus, the necessary preparations are accomplished, and we are able to set up the Hall-MHD mode filter.

We have demonstrated that for any fixed wave number $k$ six solutions of the dispersion relation exist for $\omega$ with six corresponding eigenvectors. However, for a fixed frequency $\omega$ the existence of six solutions $k$ is not guaranteed. Because of this we start with a wave number $k$ with a measured state vector $\vec{u}(k)$ and determine the eigenvectors of Eq. (12), $\vec{e}_{i}(i=1, \ldots, 6)$. These eigenvectors form a basis of the 6-dimensional state space, and we use this basis to expand $\vec{u}(k)$. One has the decomposition:

$$
\vec{u}(k)=\sum_{i=1}^{6} g_{i} \vec{e}_{i}(k)
$$



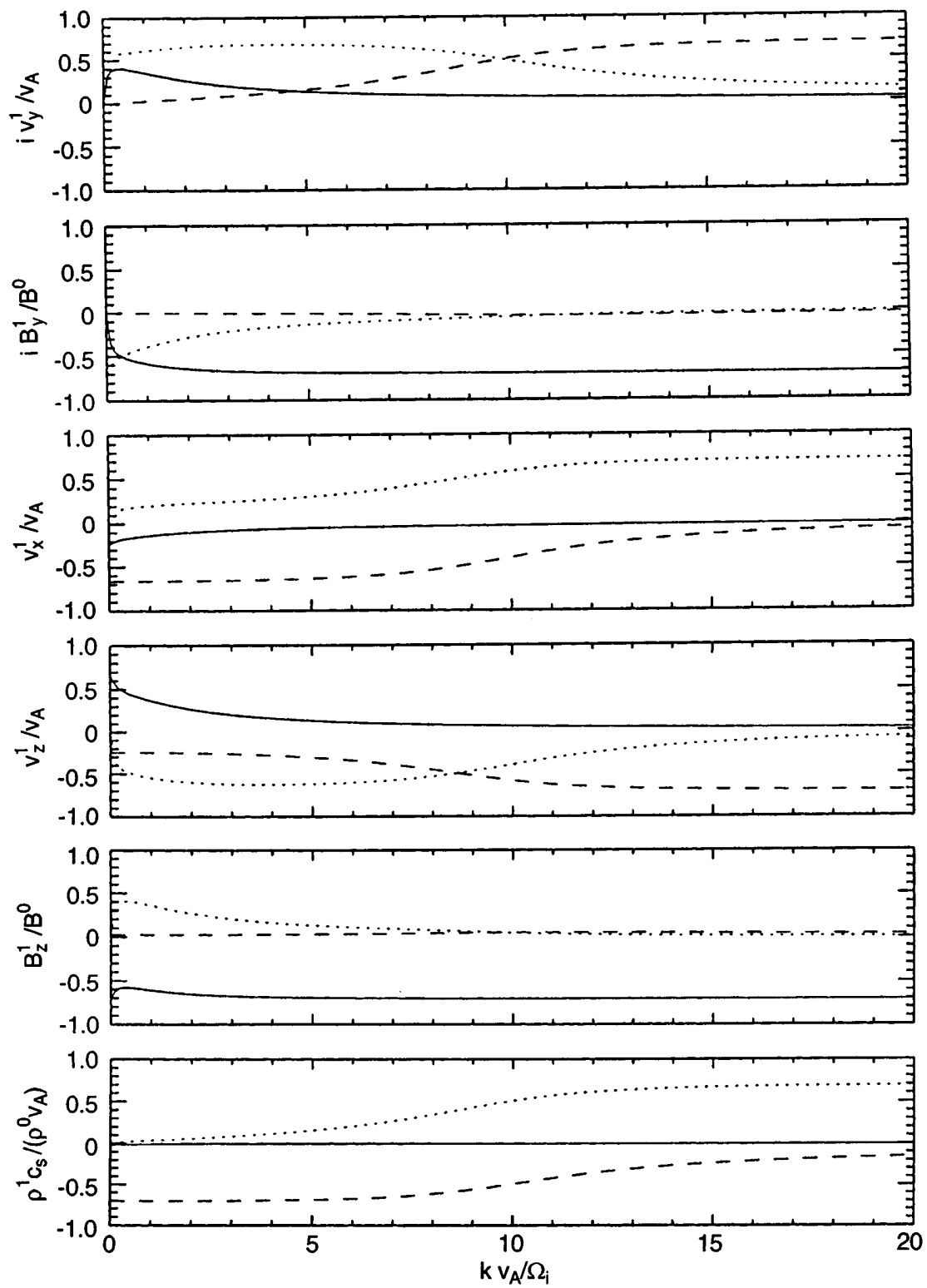

Fig. 3. The eigenvectors of Hall-MHD. Plasma background parameters: $\theta=20^{\circ}, \beta=0.01$, $v_{x}^{0}=0$. Shown are the fast mode (solid line), Alfvén mode (dotted line) and slow mode (dashed line)
The coefficients $g_{i}$ can easily be determined due to the orthogonality of the $\vec{e}_{i}$ :

$g_{i}=\vec{u} \cdot \vec{e}_{i} \quad i=1, \ldots, 6$

The total wave energy density can be calculated from the state vector $\vec{u}$ :

$w=\frac{1}{2} \rho^{0} v_{A}^{2} u^{2}$

From Eq. (9) we see that $w$ is the sum of the kinetic energy $\frac{1}{2} \rho^{0} v^{2}$, the magnetic energy $\left(B^{2} / 2 \mu_{0}\right)$ and the pressure energy $\frac{1}{2} c_{s}^{2} \frac{\left(\rho^{1}\right)^{2}}{\rho^{0}}$. Thus the relative contributions of the different modes to the total wave energy are:

$w_{i}=\frac{\left|g_{i}\right|^{2}}{\sum_{j=1}^{6}\left|g_{j}\right|^{2}}$

The mode filter is able to provide an analysis for any measured state vector $\vec{u}$, the contributions of the HallMHD modes to each particular measurement can be determined, and therefore the contributions of the various modes to the total wave energy.

\section{Test of the mode filter with simulated data}

Before the mode filter can be used, some information about the plasma has to be known. First, the plasma background parameters $\vec{B}^{0}, \rho^{0}$ and $\vec{v}^{0}$ are needed to set up the model. These are necessary to determine the dispersion and polarization of the wave modes and to obtain the system matrix $\mathbf{Z}$. The plasma background parameters can easily be determined from measurements of the temporal variations of $\vec{B}, \rho$ and $\vec{v}$ by taking their mean values. Knowledge of the direction of wave propagation is also needed to calculate the eigenmodes of the model. This direction is represented by the angle $\theta$ in Fig. 1. This $\theta$ is necessary since the dispersion and polarization characteristics of the wave modes usually depend on $\theta$ : indeed the system matrix $\mathbf{Z}$ depends on $\theta$. 
The direction of the wave propagation can be determined by a minimum variance analysis, for example. For this analysis the data from a single satellite in the plasma are sufficient.

Furthermore, the spectral amplitudes of the variations $\vec{B}^{1}, \rho^{1}$ and $\vec{v}^{1}$ have to be known. As was discussed in the previous section, a fixed value for $k$ is taken, and the amplitudes $\vec{B}^{1}(k), \rho^{1}(k)$ and $\vec{v}^{1}(k)$ have to be determined. These amplitudes can be collected in a state vector $\vec{u}(k)$ using Eq. (9). Since the wave number $k$ appears in the system matrix $\mathbf{Z}$, see Eq. (11), its value must also be known.

If the measured data are time series, the frequency $\omega$ and the spectrum with respect to $\omega$ can be estimated using a Fourier transform. The wave vector $k$ is not as easily determined. This requires a spatial resolution of the measurement. In space plasmas, this can be accomplished by a multi-satellite mission such as the CLUSTER mission and using a wave analyzer much as suggested by Motschmann et al. (1996). In laboratory plasmas, measurements at different points may be taken. In a numerical simulation, the determination of $k$ and $\vec{u}(k)$ is much easier. Here, $\vec{B}, \rho$ and $\vec{v}$ are known at any point of the simulation box, and a Fourier transform can be performed, both in space and time.

If the above information is available, the polarization vectors $\vec{e}_{i}(k)$ can be calculated and the measured state vector $\vec{u}(k)$ can be analysed using Eqs. (15) and (16). To test the mode filter it is now applied to simulated data. These data were computed using a $2 \frac{1}{2}$-D hybridcode simulation as described in Motschmann et al. (1997). In the middle of the simulation box which is sketched in Fig. 4 we place the emitting antenna radiating the assumed plasma waves. This wave source is constructed as a line along which the temporally changing values of $\vec{B}^{1}, \vec{v}^{1}$ and $\rho^{1}$ are given as boundary conditions. These values correspond to the polarization and frequency of the desired wave mode or polarizations and frequencies of a superposition of different modes. Since the antenna is oriented along the $y$-axis, waves propagating in the $x$ direction are excited.

Wave polarizations and frequencies are calculated using the Hall-MHD model by solving the eigenvalue problem in Eq. (12) for given plasma background parameters and wave number $k$. The amplitudes of the

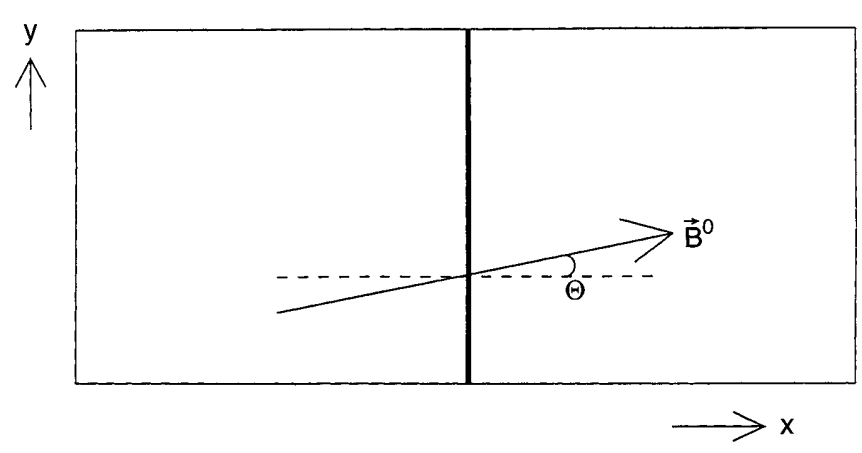

Fig. 4. The geometry of the simulation box waves are chosen as $B^{1} / B^{0}=0.05$ : that is the disturbances are small compared with the background values to ensure the validity of the linear theory.

In our simulation the antenna emits signals with the prescribed polarizations and frequencies into the model plasma which then propagate through the simulation box. After some simulation time, but before the wave front reaches the end of the simulation box, the resulting wave fields can be analysed. A portion of the simulation box between the antenna and the wave front in which the plasma waves are well developed is taken for the analysis.

Now the wave number $k$ and the state vector $\vec{u}(k)$ are to be determined. First a Fourier analysis is performed to determine the $\vec{k}$-spectrum. Since a single wave mode or different wave modes with the same wave number vector $\vec{k}$ are excited, these spectra should show only one peak at the location of this wave number. In all simulations presented here this single peak in the spectra can be clearly observed. The values of the spectral amplitudes of the parameters $\vec{B}^{1}, \vec{v}^{1}$ and $\rho^{1}$ at this peak yield the state vector $\vec{u}$ that can be analysed by the mode filter, and the wave number $k$ is known from the position of the peak. The background parameters are known too, so the mode filter can be applied.

At first, the propagation of a fast mode in the model plasma is simulated. A wave number of $k \cdot v_{A} / \Omega_{i}=0.611$ is assumed, and the plasma background parameters are chosen as:

$\theta=20^{\circ}, \quad \beta=10^{-4}, \quad v_{x}^{0}=0$

The frequency of the wave is $\omega=0.7944 \cdot \Omega_{i}$, which excludes the use of an MHD mode filter. The analysis extends over a region of the simulation box that lies "right" of the emitting antenna, so that a forward propagating wave has to be registered. Figure 5 shows the power spectrum of the component $B_{y}^{1}$ in this region. It exhibits a single peak, as expected. The position of the peak indicates that the $y$-component of $\vec{k}$ vanishes, the wave propagation is directed along the $x$-axis, as it is to expect from the geometry of the simulation box. This peak can be found in the spectra of the other components as well. The location of the peak is $k \cdot v_{A} / \Omega_{i}=0.626$. Considering the spatial discretization of the simulation box this result coincides with the value used for the calculation of the frequency and polarization of the wave.

The mode filter yields the following result for the forward propagating wave:

$$
\begin{array}{cccccc}
w_{F,+} & w_{F,-} & w_{A,+} & w_{A,-} & w_{S,+} & w_{S,-} \\
0.9997 & 0.0000 & 0.0002 & 0.0000 & 0.0000 & 0.0001
\end{array}
$$

This table shows the relative contributions $w$ of the fast $(\mathrm{F})$, Alfvén (A) and slow mode (S), each forward $(+)$ and backward (-) propagating, to the total wave energy. The Hall-MHD mode filter assigns more than $99.9 \%$ of the wave energy to the correct mode, the forward propagating fast mode. This demonstrates the power of the filter, at least when applied to simulated data. 


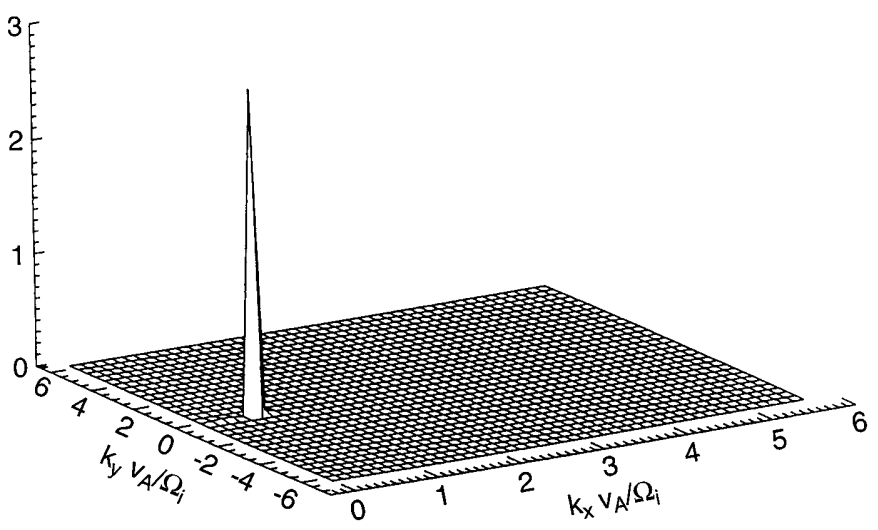

Fig. 5. The power spectrum of $B_{y}$ in arbitrary units

It is of interest to check if the mode filter is able to analyse a wave field consisting of different modes and to find the right contributions to the total wave energy. This situation is tested using a superposition of a fast and an Alfvén mode excited in the model plasma. The amplitudes are chosen in such a way that the ratio of the wave energies of the fast and Alfvén mode is $2: 1$. The frequencies of the two wave modes are $\omega_{F}=0.7944 \cdot \Omega_{i}$ and $\omega_{A}=0.4416 \cdot \Omega_{i}$. The filter result of this simulation is:

$\begin{array}{cccccc}w_{F,+} & w_{F,-} & w_{A,+} & w_{A,-} & w_{S,+} & w_{S,-} \\ 0.6879 & 0.0033 & 0.3085 & 0.0001 & 0.0001 & 0.0001\end{array}$

Again excellent agreement between the simulated wave parameters and those resulting from the mode filter analysis is achieved. There is only a minor deviation, that is the contribution of the fast mode is a little too high. This can be explained by the higher phase velocity of the fast mode. After the start of the simulation, this mode propagates faster through the simulation box, so that the fast mode is a little better developed in the region that is analysed.

The mode filter is based on a linear theory, which implies that the applicability of this filter is limited to low amplitudes of the plasma waves. To estimate how "low" the amplitudes need to be to allow a useful and practical application of the mode filter, a series of simulations with rising amplitudes is performed. The numerical code used here is able to handle nonlinear plasma waves. The propagation of an Alfvén mode is simulated, the plasma background parameters are kept unchanged except the plasma $\beta$ which is raised to 0.1 for this series as higher amplitudes lead to numerical instabilities for the very low $\beta$ that was used in the previous simulations.

The series of simulations starts with a low amplitude for which the mode filter yields good results. Then the amplitude is raised from simulation to simulation. If the mode filter assigns wave energy to other modes, this is regarded as a sign for the beginning breakdown of the linear theory, with the limit of the applicability of the mode filter being reached. The frequency of the Alfvén mode is $\omega=0.4451 \cdot \Omega_{i}$. The series of simulations is started with an amplitude of $B^{1} / B^{0}=0.12$ for which the mode filter assigns more than $99 \%$ of the wave energy to the Alfvén mode. The limit for the applicability of the mode filter is reached at amplitudes $B^{1} / B^{0} \approx 0.3$. For higher amplitudes, application of the mode filter ceases with less than $95 \%$ of the wave energy being assigned to the Alfvén mode.

This amplitude of $B^{1} / B^{0} \approx 0.3$ is however only a rough estimate for the limit of the applicability of the mode filter based on a linear theory. A similar series of simulations of the fast mode showed no sign of a deterioration of the function of the mode filter. So this limit depends on the wave mode and probably also on the plasma background parameters. There may be cases in which the limit is even less than $B^{1} / B^{0} \approx 0.3$.

We conclude that the Hall-MHD mode filter yields very good results in analysing the simulated data. The correct modes are clearly identified with correct contributions of different modes to the total wave energy having been determined. The simulations with rising amplitudes show that this mode filter may be used for wave amplitudes up to the order of $B^{1} / B^{0} \approx 10^{-1}$.

\section{MHD versus Hall-MHD mode filter}

Application of the Hall-MHD mode filter requires knowledge of the wave vector $\vec{k}$, while for application of the MHD mode filter only the direction of wave propagation needs to be known. Thus, despite its increased frequency range the Hall-MHD mode filter is more difficult to use. Consequently we have studied which errors may occur if the MHD mode filter is applied to situations where use of the Hall-MHD mode filter is required. Such a study allows us to develop a rule to select in which situation either of the mode filters may be used.

We have constructed the polarizations of various plasma wave modes using the Hall-MHD model. Using these modes as input for the Hall-MHD mode filter would result in the detection of pure modes. Using these as input for the MHD mode filter would give us expansion coefficients different from using the HallMHD filter. Deviations of the expansion coefficients may thus be used as a measure of the applicability of the MHD mode filter.

To set up the system matrix $\mathbf{Z}$, we have to choose plasma background parameters. We take a plasma consisting of protons and electrons and the following background:

$\theta=20^{\circ}, \quad \beta=0.01, \quad v_{x}^{0}=0$

First a very small wave number $k$ is chosen: $k \cdot v_{A} / \Omega_{i}=0.00611$. The frequencies of the fast, Alfvén and slow mode are:

$\omega_{F}=0.0061 \cdot \Omega_{i}, \quad \omega_{A}=0.0057 \cdot \Omega_{i}, \quad \omega_{S}=0.0006 \cdot \Omega_{i}$

The application of the MHD mode filter to the HallMHD polarization vector of the forward propagating fast mode yields:

$\begin{array}{cccccc}w_{F,+} & w_{F,-} & w_{A,+} & w_{A,-} & w_{S,+} & w_{S,-} \\ 0.9978 & 0.0000 & 0.0022 & 0.0000 & 0.0000 & 0.0000\end{array}$


The analysis of the Alfvén mode leads to:

$\begin{array}{cccccc}w_{F,+} & w_{F,-} & w_{A,+} & w_{A,-} & w_{S,+} & w_{S,-} \\ 0.0022 & 0.0000 & 0.9978 & 0.0000 & 0.0000 & 0.0000\end{array}$

And the slow mode is decomposed in this way:

$\begin{array}{cccccc}w_{F,+} & w_{F,-} & w_{A,+} & w_{A,-} & w_{S,+} & w_{S,-} \\ 0.0000 & 0.0000 & 0.0000 & 0.0000 & 1.0000 & 0.0000\end{array}$

The MHD filter clearly detects the proper modes with only small fractions of the total wave energy being assigned to other modes.

Now a higher wave number $k$ is used: $k \cdot v_{A} / \Omega_{i}=$ 0.611. The frequencies of the different modes are:

$\omega_{F}=0.794 \cdot \Omega_{i}, \quad \omega_{A}=0.442 \cdot \Omega_{i}, \quad \omega_{S}=0.057 \cdot \Omega_{i}$

The frequencies of the fast and Alfvén modes are still less than the ion cyclotron frequency, but they are not much smaller as before. The analysis of the Hall-MHD polarization vectors by the MHD mode filter yields:

fast mode:

$\begin{array}{cccccc}w_{F,+} & w_{F,-} & w_{A,+} & w_{A,-} & w_{S,+} & w_{S,-} \\ 0.5412 & 0.0092 & 0.4381 & 0.0114 & 0.0001 & 0.0000\end{array}$

Alfvén mode:

$\begin{array}{cccccc}w_{F,+} & w_{F,-} & w_{A,+} & w_{A,-} & w_{S,+} & w_{S,-} \\ 0.4382 & 0.0113 & 0.5411 & 0.0092 & 0.0001 & 0.0001\end{array}$

slow mode:

$\begin{array}{cccccc}w_{F,+} & w_{F,-} & w_{A,+} & w_{A,-} & w_{S,+} & w_{S,-} \\ 0.0000 & 0.0000 & 0.0001 & 0.0001 & 0.9998 & 0.0000\end{array}$

The table shows that the MHD filter is not able to detect the fast and Alfvén mode, but superpositions of these two modes are detected. The slow mode, however, is not affected at all because its frequency is still small compared with $\Omega_{i}$. As real data may contain superpositions of fast and Alfvén modes, the study indicates that the condition $\omega \ll \Omega_{i}$ needs to be verified carefully before the MHD mode filter is used. Otherwise the Hall-MHD mode filter should be the preferred method to determine wave energy contributions to particular modes.

Applicability of both mode filters, MHD and HallMHD filter, also depends on the numerical values of the system matrix $\mathbf{Z}$. Errors in determining the plasma background parameters could affect the results of these filters. To check this, a similar analysis to the above comparison between MHD and Hall-MHD has been performed. The polarizations of the Hall-MHD modes have been calculated for a given set of plasma background parameters, and the resulting state vectors have been analysed by a Hall-MHD mode filter with background parameters differing from the real values used. Deviations of the plasma background parameters of up to $25 \%$ from the original values lead to errors of approximately $1 \%$ in the determination of the contributions of the different modes to the total wave energy. Thus we conclude that the mode filter is very robust against errors in determining the plasma background parameters.

\section{Entropy waves and tangential structures}

The mode filter is able to analyse any Hall-MHD signal with spectral peak power at given wave number $k$. The results obtained are unique and there are no residuals, that is the state vector is completely decomposed into the contributions of the different modes. A full error analysis is an enormous task, but a finite signal-to-noise ratio of the measurement enters the determination of the state vector $\vec{u}(k)$ and thus influences the contributions to the total wave energy assigned to the different wave modes. Thus, the signal-to-noise ratio limits the accuracy of the mode filter.

The question arises what happens if a phenomenon is observed which cannot be described by the underlying plasma model, such as the passage of a satellite through a stationary structure in the plasma. In the satellite frame the movement through a spatially periodic, stationary structure would be recognized as a wave passing by. In these cases one could suspect that the mode filter misinterpretates the observations as HallMHD waves.

This illustrates that the assumptions of the HallMHD have to be checked carefully: The plasma background system has to be homogeneous, the frequencies considered have to be much smaller than the lower hybrid frequency, and the temperature has to be low enough to justify the neglect of kinetic effects in the Hall-MHD. For a detailed comparison between HallMHD and kinetic waves see Krauss-Varban et al. (1994). Furthermore, the observed wave trains have to be long enough, since the Fourier transform performed in the development of the mode filter corresponds to a plane wave setup, and the amplitude of the signal has to be small enough to render linear theory applicable.

If these conditions are not fulfilled, the Hall-MHD mode filter may yield erroneous results. It is always possible to calculate the polarization vectors of the HallMHD model which form a basis of the state space, so any measured state vector can be developed into this basis. But if the Hall-MHD model is not valid, these Hall-MHD polarization vectors deviate from the real polarization vectors of the corresponding wave modes. Thus, projecting the measured state vector on the eigenvectors of the Hall-MHD model yields wrong contributions of the modes to the total wave energy.

The comparison between Hall-MHD and MHD mode filter in Sect. 4 illustrates this effect for the MHD mode filter. If the MHD condition $\omega \ll \Omega_{i}$ is not met, the polarization vectors of the MHD model differ largely from the vectors yielded by the Hall-MHD model which is still valid, leading to large errors of the MHD mode filter. Violating the Hall-MHD conditions will cause errors of the Hall-MHD mode filter in the same way. Furthermore, additional wave modes may exist not modelled by the Hall-MHD. Interpreting their polarizations as combinations of Hall-MHD modes is senseless, of course.

Thus, care has to be taken that the above Hall-MHD conditions are valid, the mode filter can be used only within the scope of its underlying plasma model. 
Our basic Eqs. (1)-(3) neglect any changes of the entropy $s$ which is treated as temporally and spatially constant. Therefore, entropy waves are structures which cannot be described by the Hall-MHD as used here. Regarding entropy as a further parameter describing variations in the plasma adds another equation to the basic Eqs. (1)-(3): the adiabatic equation $\mathrm{d} s / \mathrm{d} t=0$. With this equation the dimension of the state space is expanded by the entropy coordinate to seven, and since the eigenvalue problem becomes 7-dimensional, a new wave mode exists: the entropy wave. The polarization of this entropy wave can be described following Akhiezer et al. (1975) using a 2-dimensional state vector

$\tilde{\vec{u}}=\left(\rho^{1}, s^{1}\right)^{T}$

with the following polarization vector:

$\vec{e}_{7}=\left(-\left(\frac{\partial p}{\partial \rho}\right)_{s},\left(\frac{\partial p}{\partial s}\right)_{\rho}\right)^{T}$

From this it follows that the entropy wave only consists of a variation of the density and entropy, which may also be described as a variation of the density and temperature. The pressure $p$ stays constant throughout this variation. The phase velocity of the entropy wave vanishes in the plasma rest frame, that is the entropy wave is a stationary structure. This variation does not need to have a special direction or wave number. If there is a spatially periodic entropy variation with the wave number $k$, and if a satellite crosses it with a speed of $v_{x}^{0}$, then the resulting measurement might be given to the mode filter as input state vector.

The mode filter presented here only takes into account the variation of the density $\rho$ when the entropy wave is crossed. Thus the filter interprets any crossing of an entropy wave as a superposition of the six Hall-MHD wave modes where the contributions to the other parameters $\vec{v}^{1}$ and $\vec{B}^{1}$ cancel each other. This erroneous interpretation can be avoided using the frequency information available. The measured data usually consist of time series, and a Fourier-analysis yields the spectrum. If the satellite crosses with a speed of $v_{x}^{0}$ a periodic structure with wave number $k$, the spectrum would show peak power at $\omega=k \cdot v_{x}^{0}$. However, if the interpretation as a superposition of 6 Hall-MHD modes would be correct, the spectrum should exhibit six power spectral peaks at the respective frequencies of these modes in the satellite frame reference. In this way a resulting decomposition of the mode filter can be checked for false results based on the presence of entropy waves.

The entropy wave is a stationary structure in the plasma. The question arises whether there are any other stationary structures within the scope of Hall-MHD, which may lead to misinterpretations by the mode filter. To examine this, we look for stationary solutions of the basic Eqs. (1)-(3). If these basic equations for the plasma rest frame are written in components using the coordinate system presented earlier in this paper and under consideration of the stationarity condition $\partial / \partial t \equiv 0$, one can easily find that any disturbances $\vec{v}^{1}$, $\vec{B}^{1}$ and $\rho^{1}$ can only exist if $\cos \theta=0 \Leftrightarrow \theta=90^{\circ}$.
In this case, the background magnetic field $\vec{B}^{0}$ points in the $z$-direction. So we can say that stationary structures have to be tangential structures. Furthermore, Eq. (2) yields $\vec{j}^{1} \times \vec{B}^{0}=\nabla p^{1}$, such that the Hall-term $\frac{1}{e N^{0}} \nabla \times\left(\vec{j}^{1} \times \vec{B}^{0}\right)$ is equal to zero, since $\nabla \times \nabla p=0$. There is no difference between MHD and Hall-MHD in this discussion of stationary structures. This can also be deduced from the structure of the system matrix $\mathbf{Z}$ in Eq. (11). Both Hall-terms contain the factor $\cos \theta$, and vanish here.

The solutions of the stationary basic equations are independent variations of $v_{y}^{1}, v_{z}^{1}$ and $B_{y}^{1}$ and a variation of the pressure $p^{1}$ and $B_{z}^{1}$ so that the sum of kinetic and magnetic pressure $p+\left(B^{2} / 2 \mu_{0}\right)$ stays constant.

Only the fast mode can propagate for $\theta=90^{\circ}$, the other MHD-modes, the Alfvén and slow modes, can't (their phase velocities tend to 0 for $\theta \rightarrow 90^{\circ}$ ). A disappearing phase velocity $v_{p h}=\omega / k$ means that $\omega \rightarrow 0$ for a finite wave number $k$. So it corresponds to a stationary structure.

For the polarizations of the MHD modes as they are given in (Glassmeier et al., 1995) the limit $\theta \rightarrow 90^{\circ}$ can be calculated. The polarizations of the Alfvén and slow mode, whose phase velocities are 0 , correspond to the solutions of the stationary basic equations: The forward and backward propagating Alfvén waves lead to the variations of $v_{y}^{1}$ and $B_{y}^{1}$, the slow modes yield the variations of $v_{z}^{1}$ and the kinetic and magnetic pressure.

These calculated polarization vectors correspond to the eigenvectors which result from the eigenvalue problem [Eq. (12)] in the case presented here $\left(v_{x}^{0}=0\right.$, $\cos \theta=0)$. So all solutions of the stationary basic equations can be described by the polarization vectors which result from the eigenvalue problem on which the mode filter is based. This means that the mode filter is able to handle the signatures of stationary structures.

From this it follows that the mode filter is able to analyse the signals resulting from the passage of the satellite through a stationary structure. Due to the satellite's speed the system matrix $\mathbf{Z}$ now contains a plasma flow component $v_{x}^{0} \neq 0$, but from the structure of $\mathbf{Z}$ as it is given in Eq. (11) it follows that this $v_{x}^{0}$ is only a constant which is added to the eigenvalues, and it doesn't affect the eigenvectors (see also Appendix). So the movement of the satellite has no effect on the function of the mode filter.

Thus we may conclude that there is no danger of erroneous analyses when a stationary structure, as it is described here, is traversed.

\section{Conclusions and summary}

The plasma model of MHD is restricted to low frequencies $\omega \ll \Omega_{i}$, and so is the MHD mode filter. The extension of this mode filter to higher frequencies requires a generalization of the plasma model.

The use of the Hall-MHD instead of the MHD relaxes the frequency limit $\omega \ll \Omega_{i}$. The Hall-MHD is only limited by $\omega \ll \omega_{L H}$, which is a rather large 
expansion of the frequency range since $\omega_{L H} \approx$ $\sqrt{m_{i} / m_{e}} \cdot \Omega_{i}$. A mode filter based on the Hall-MHD is constructed analogous to the MHD mode filter. The spectral amplitudes of the magnetic field, bulk velocity and density are collected in a state vector, and any measured state vector is decomposed into the contributions of the different wave modes in a unique way. In contrast to the MHD case, the Hall-MHD mode filter requires not only the knowledge of the plasma background parameters but also of the wave number $k$, as the Hall-MHD modes display dispersion. A precise determination of $k$ may be realized by multi-point measurements as it is foreseen in the Cluster II mission.

The range of validity of the Hall-MHD has no limit at low frequencies. In the limit $\omega \rightarrow 0$, MHD and HallMHD coincide. The mode filters based on these plasma models operate on the same state space, and therefore these filters also coincide in the limit $\omega \rightarrow 0$.

Thus at $\omega \approx 0$ a direct comparison of their results is possible. For frequencies $\omega \approx \Omega_{i}$, however, the use of the MHD can lead to large errors in determining the composition of plasma wave data. This demonstrates that the mode filter can only be applied when its underlying plasma model is valid. This applies as well to the Hall-MHD mode filter, the assumptions of the HallMHD have to be checked carefully before the mode filter is used.

An application of the Hall-MHD mode filter to simulated plasma wave fields shows its ability to identify the modes excited in the simulation box. If more than one wave mode is present in the model plasma the correct energetic contributions are assigned to the individual wave modes.

As the basic equations of the Hall-MHD were used in a linear approach the mode filter is only able to handle linear plasma waves, i.e. waves with small amplitudes. A series of simulations with rising amplitudes showed a limit of $B^{1} / B^{0} \approx 0.3$ for the applicability of the mode filter. Thus the linear theory is restricted to amplitudes up to the order of $B^{1} / B^{0} \approx 10^{-1}$.

Extension of the mode filter to frequencies $\omega \approx \omega_{L H}$ or even higher requires a further generalization of the plasma model, e.g. a multi-fluid-theory without any frequency limit, or even a kinetic approach. The dimension of the state space is strongly increased and therefore these approaches will be the subject of future work.

Since the development of a mode filter, like the one presented here, starts with the basic equations of the plasma model, the mode filter can only detect those plasma wave modes which are described by the underlying plasma model. In general, only those wave modes can be found that are searched for.

\section{Appendix}

\section{The eigenvalues of the system matrix $\mathbf{Z}$}

In this appendix the derivation of the analytic solutions $\omega(k)$ of the dispersion relation of the Hall-MHD is shown in more detail. It has already been discussed that solving the dispersion relation corresponds to finding the eigenvalues of the system matrix $\mathbf{Z}$ as it is given in Eq. (11).

The eigenvalues of a matrix are determined by finding the zeroes of its characteristic polynomial. For the system matrix $\mathbf{Z}$ it follows from the eigenvalue Eq. (10) that the eigenvalues correspond to the phase velocities $\omega / k$ and the eigenvectors to the polarizations of the different wave modes. The matrix $\mathbf{Z}$ is a function of the wave number $k$, and so the eigenvalues and eigenvectors will depend on $k$, too.

Since the matrix $\mathbf{Z}$ is 6-dimensional, its characteristic polynomial has the degree 6 . The structure of the system matrix $\mathbf{Z}$ and the calculation of the characteristic polynomial appears to indicate that it is not possible to write this polynomial as a product of polynomials with lower degrees. There is no analytic formula for the zeroes of a polynomial of the degree 6 , and so it appears to be impossible to find analytic expressions for the 6 eigenvalues of $\mathbf{Z}$.

However, in case of a plasma at rest $\left(v_{x}^{0}=0\right)$, the characteristic polynomial of $\mathbf{Z}$ contains only even powers of $\omega / k$. So this polynomial can be regarded as a cubic polynomial in $(\omega / k)^{2}$. The formula of Cardani which yields the zeroes of a cubic polynomial then allows the determination of analytic expressions for the eigenvalues of the system matrix for $v_{x}^{0}=0$.

The assumption $v_{x}^{0}=0$ seems to be a strong restriction for the analytic solution of the eigenvalue equation, but this is not the case. For a moving plasma analytic expressions for the eigenvalues can also be found.

This can be seen from the structure of the system matrix $\mathbf{Z}$ as it is given in Eq. (11). The background plasma flow speed $v_{x}^{0}$ occurs only on the principal diagonal of $\mathbf{Z}$. The matrix can be decomposed in this way:

$\mathbf{Z}=\tilde{\mathbf{Z}}+v_{x}^{0} \cdot \mathbf{1}_{6}$

Here $\tilde{\mathbf{Z}}$ is the system matrix for $v_{x}^{0}=0$ and $\mathbf{1}_{6}$ the 6dimensional unity matrix.

This decomposition of the system matrix $\mathbf{Z}$ enables us to determine its eigenvalues. If $\frac{\omega}{k}$ is an eigenvalue of $\tilde{\mathbf{Z}}$ and $\vec{u}$ the corresponding eigenvector, then:

$$
\begin{aligned}
& \tilde{\mathbf{Z}} \cdot \vec{u}=\frac{\omega}{k} \vec{u} \Leftrightarrow \tilde{\mathbf{Z}} \cdot \vec{u}+v_{x}^{0} \cdot \vec{u}=\left(\frac{\omega}{k}+v_{x}^{0}\right) \vec{u} \Leftrightarrow \\
& \mathbf{Z} \cdot \vec{u}=\left(\frac{\omega}{k}+v_{x}^{0}\right) \vec{u}
\end{aligned}
$$

So $\mathbf{Z}$ and $\tilde{\mathbf{Z}}$ have the same eigenvectors, and thus the polarizations of the wave modes are independent of the plasma flow speed. The eigenvalues which represent their phase velocities are yielded for a moving plasma simply by adding the flow speed component $v_{x}^{0}$ to the values calculated in the plasma rest frame. This is simply Doppler shifting of the frequencies of the waves.

So the eigenvalues of the system matrix $\mathbf{Z}$ for a moving plasma can be easily obtained from the solutions for the plasma rest frame $v_{x}^{0}=0$.

These solutions for the plasma rest frame are now determined. For $v_{x}^{0}=0$ the characteristic polynomial of $\mathbf{Z}$ has, as already mentioned, the degree 3 in $(\omega / k)^{2}$, and analytic expressions for its zeroes can be found.

But before these solutions are presented, some abbreviations have to be introduced: 


$$
\begin{aligned}
X 1 & :=c_{s}^{2}+v_{A}^{2}+\cos ^{2} \theta v_{A}^{2}+\cos ^{2} \theta d^{2} k^{2} v_{A}^{2} \\
X 2 & :=2 c_{s}^{2}+c_{s}^{2} d^{2} k^{2}+v_{A}^{2} \\
a & :=-X 1^{2}+3 \cos ^{2} \theta v_{A}^{2} X 2 \\
b & :=27 \cos ^{4} \theta c_{S}^{2} v_{A}^{4}+2 X 1^{3}-9 \cos ^{2} \theta v_{A}^{2} X 1 X 2
\end{aligned}
$$

Using these abbreviations, the following results in the plasma rest frame can be obtained using the formula of Cardani:

$$
\begin{aligned}
\left(\frac{\omega^{2}}{k^{2}}\right)_{1}= & \frac{X 1}{3}-\frac{2^{\frac{1}{3}} a}{3\left(b+\sqrt{4 a^{3}+b^{2}}\right)^{\frac{1}{3}}} \\
& +\frac{\left(b+\sqrt{4 a^{3}+b^{2}}\right)^{\frac{1}{3}}}{3 \cdot 2^{\frac{1}{3}}} \\
\left(\frac{\omega^{2}}{k^{2}}\right)_{2}= & \frac{X 1}{3}+\frac{(1+i \sqrt{3}) a}{3 \cdot 2^{\frac{1}{3}}\left(b+\sqrt{4 a^{3}+b^{2}}\right)^{\frac{1}{3}}} \\
\left(\frac{\omega^{2}}{k^{2}}\right)_{3}= & \frac{X 1}{3}+\frac{(1-i \sqrt{3})\left(b+\sqrt{4 a^{3}+b^{2}}\right)^{\frac{1}{3}}}{3 \cdot 2^{\frac{1}{3}}\left(b+\sqrt{4 a^{3}+b^{2}}\right)^{\frac{1}{3}}} \\
& -\frac{(1+i \sqrt{3})\left(b+\sqrt{4 a^{3}+b^{2}}\right)^{\frac{1}{3}}}{6 \cdot 2^{\frac{1}{3}}}
\end{aligned}
$$

These expressions seem to be complex. But the system matrix $\mathbf{Z}$ is Hermitian, so its eigenvalues are real. Using this, we can show that $4 a^{3}+b^{2} \leq 0$ and consequently $a \leq 0$. The imaginary parts in Eqs. (27)-(29) cancel each other, and these expressions can be transformed into a real form:

$$
\begin{aligned}
& \frac{\omega}{k} \sqrt{\frac{X 1}{3}+\frac{2}{3} \sqrt{-a} \cos \left(\frac{1}{3} \arctan \left(\sqrt{-1-\frac{4 a^{3}}{b^{2}}}\right)+\frac{2 m}{3} \pi\right)}, \\
& m=0,1,2
\end{aligned}
$$

The eigenvalues of the system matrix $\mathbf{Z}$ for a moving plasma $\left(v_{x}^{0} \neq 0\right)$ are simply given by:

$$
\begin{array}{r}
\frac{\omega}{k}=v_{x}^{0} \\
\pm \sqrt{\frac{X 1}{3}+\frac{2}{3} \sqrt{-a} \cos \left(\frac{1}{3} \arctan \left(\sqrt{-1-\frac{4 a^{3}}{b^{2}}}\right)+\frac{2 m}{3} \pi\right)}, \\
m=0,1,2
\end{array}
$$

The double sign denotes the forward and backward propagating wave modes. A positive and negative value for $\omega / k$ occurs because the zeroes of a polynomial in $(\omega / k)^{2}$ were determined.

The complex structure of these solutions together with the abbreviations introduced above prevents the finding of analytic expressions for the eigenvectors corresponding to these eigenvalues. So the polarizations of the Hall-MHD modes have to be calculated numerically.

Acknowledgements. This work was financially supported by DARA (now DLR). Contributions by U. M. were supported by the Deutsche Forschungsgemeinschaft through a Werner-Heisenberg-Stipendium. The authors are grateful to H. Kafemann for providing the simulated test data.

The Editor in chief thanks J. D. Huba and T. I. Woodward for their help in evaluating this paper.

\section{References}

Akhiezer, I. A., I. A. Akhiezer, R. V. Polovin, A. G. Sitenko, and K. N. Stepanov, Plasma electrodynamics, vol. 1: linear theory, Pergamon Press, Oxford, 1975.

Glassmeier, K.-H., U. Motschmann, and R. V. Stein, Mode recognition of MHD wave fields at incomplete dispersion measurements, Ann. Geophys., 13, 76-83, 1995.

Gurnett, D. A., S. Shawhan, N. M. Brice, and R. L. Smith, Ion cyclotron whistlers, J. Geophys. Res., 70, 1665-1668, 1965.

Hassam, A. B., and J. D. Huba, Magnetohydrodynamic equations for systems with large larmor radius, Phys. Fluids, 31, 318-325, 1988.

Holter, Ø. and A. Kildal, Waves in plasmas, in: A. Egeland, Ø. Holter, A. Omholt (eds.), Cosmical geophysics, chapter 18, Universitetsforlaget, Oslo, 1973.

Huba, J. D., Theory and simulation of a high-frequency magnetic drift wave. Phys. Fluids B, 3, 3217-3225, 1991.

Huba, J. D., Hall magnetohydrodynamics in space and laboratory plasmas, Phys.Plasmas, 2, 2504-2513, 1995.

Kingsep, A. S., K. V. Chukbar, and V. V. Yan'kov, Electron magnetohydrodynamics, in: B. B. Kadomtsev (ed.), Reviews of plasma physics, vol. 16, 243, Consultants Bureau, New York, 1990.

Krauss-Varban, D., N. Omidi, and K. B. Quest, Mode properties of low frequency waves: kinetic theory versus Hall-MHD, J. Geophys.Res., 99, 5987-6009, 1994.

Motschmann, U., T. I. Woodward, K.-H. Glassmeier, D. J. Southwood, and J.-L. Pinçon, Wavelength and direction filtering by magnetic measurements at satellite arrays: generalized minimum variance analysis, J. Geophys. Res, 101, 4961-4965, 1996.

Motschmann, U., H. Kafemann, and M. Scholer, Nongyrotropy in magnetoplasmas: simulation of wave excitation and phasespace diffusion, Ann. Geophys., 15, 603-613, 1997.

Samson, J. C., Pure states, polarized waves and principal components in the spectra of multiple geophysical time-series, Geophys. J. R. Astr. Soc., 72, 647-664, 1983.

Samson, J. C. and J. V. Olson, Some comments on the descriptions of the polarisation states of waves, Geophys. J. R. Astr. Soc., 61, 115-129, 1980.

Siscoe, G. L., Solar system magnetohydrodynamics, in: R. L. Carovillano and J.M. Forbes (eds.), Solar-terrestrial physics, 11-100, D. Reidel, Dordrecht, 1983.

Spitzer, L. Jr., Physics of fully ionized gases, Wiley, New York, 1962.

Stix, T. H., The theory of plasma waves, McGraw-Hill, New York, 1962.

Swanson, D. G., Plasma waves, Academic Press, Boston, 1989. 